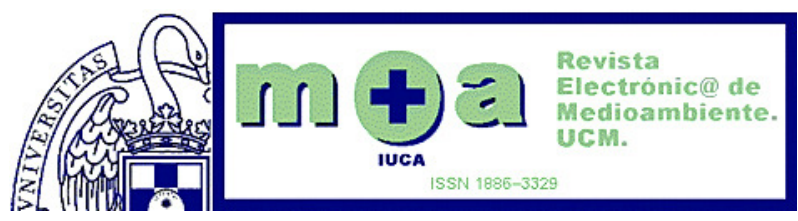

\title{
ANÁLISIS DE LA CALIDAD GENERAL DEL AGUA SUPERFICIAL EN LA CUENCA HIDROGRÁFICA DEL JÚCAR: PERIODO 2000-2009
}

\author{
E. BEAMONTE CÓRDOBA \\ Profesor Titular del Departamento de Economía Aplicada de la Universidad de Valencia \\ beamonte@uv.es
}

\section{A. CASINO MARTÍNEZ}

Catedrático de Escuela Universitaria del Departamento de Economía Aplicada de la Universidad de Valencia Alejandro.Casino@uv.es

\section{E. J. VERES FERRER}

Profesor Titular del Departamento de Economía Aplicada de la Universidad de Valencia Ernesto.Veres@uv.es

Recibido: 01-02-2012

Aceptado: $15-06-2012$

\section{RESUMEN}

En este trabajo se analiza la calidad del agua superficial en el ámbito de la Confederación Hidrográfica del Júcar, a lo largo del periodo 2000-2009. En primer lugar, se determinan los correspondientes valores del denominado Índice de Calidad General (ICG), en un amplio conjunto de puntos de muestreo y en distintos momentos del tiempo, a partir de los datos observados sobre los parámetros físico-químicos que intervienen en dicho índice. Con los resultados obtenidos se evalúa la calidad del agua superficial, independientemente de su posible uso. Posteriormente, utilizando métodos de análisis de datos de panel, se modeliza el comportamiento del índice general en función de sus parámetros más importantes y otras variables de control. Esta metodología permite tratar el efecto individual específico de cada uno de los puntos de muestreo seleccionados con más flexibilidad que los métodos clásicos de regresión.

Palabras clave: calidad del agua, Confederación Hidrográfica del Júcar, ICG, estaciones de control 


\title{
General quality of surface water analysis in the Júcar watershed: 2000-2009 period
}

\begin{abstract}
This paper analyzes the quality of surface water in the Confederación Hidrográfica del Júcar, over the period 2000-2009. First, in a wide range of sampling points and at different times, the values for the General Quality Index (GQI) have been determined from observed data on the physical and chemical parameters defining the index. The results have allowed to evaluate the quality of surface water, regardless of their possible use. Subsequently, the study has been carried out to model the behavior of the general index based on their most important parameters and other control variables, by using panel data analysis. This methodology allows to consider the specific individual effect of each sampling point with more flexibility than classical regression methods.
\end{abstract}

Keywords: water quality, Confederación Hidrográfica del Júcar, ICG, control stations

\section{Analyse de la qualité globale des eaux superficielles à le bassin du Júcar: période 2000-2009}

\section{RÉSUMÉ}

Ce document analyse la qualité des eaux superficiels dans le domaine de la Confederación Hidrográfica del Júcar (L'Espagne), dans la période 2000-2009. D'abord, nous déterminons les valeurs qui correspondent au l'index de qualité générale (IQG), pour un grand nombre de points d'échantillonnage et pour différents moments du temps, en utilisant des données observées sur les paramètres physico-chimiques impliqués dans cet index. Avec les résultats obtenus, nous procédons à évaluer la qualité des eaux superficiels, indépendamment de leur utilisation possible. Depuis, en employant des méthodes d'analyse des données de panel, le comportement de l'index global est modélisé comme une fonction de ses paramètres les plus importants et des autres variables de contrôle. Cette méthodologie permet analyser mieux les effets spécifiques individuels des différents points d'échantillonnage sélectionnés, que les méthodes traditionnelles de régression.

Mots-clés: qualité de l'eau, Confederación Hidrográfica del Júcar, ICG, stations de contrôle

\section{INTRODUCCIÓN}

La calidad del agua es un factor importante a tener en cuenta a la hora de valorar el desarrollo sostenible de una determinada zona. En los últimos años, el creciente interés por su adecuada medición se ha puesto de 
manifiesto en diversos estudios (Beamonte et al., 2004, 2005, 2007 y 2010; Prat y Munné, 2000; Sánchez et al., 2007; Villalba et al., 1995). Asimismo, la consideración de los aspectos económicos relacionados con el agua y su calidad, también ha conducido a la realización de un gran número de investigaciones (Barberán et al., 2008; Barreiro y Pérez, 2006; Bergstrom et al., 2001; González, 2005; Júdez et al., 2001; Otto y Holtkamp, 1999; Pérez et al., 2010; Ribaudo, 2003; Sevilla et al., 2010; Steinnes, 1992).

La evaluación de la calidad del agua, que depende del uso que se vaya a hacer de ella, se efectúa en base a la medición de diferentes parámetros. La integración, en un único índice sintético, de los valores observados sobre el correspondiente conjunto de parámetros se puede realizar mediante la aplicación de distintos indicadores. El Ilamado Índice de Calidad General (ICG) es muy utilizado por las distintas Confederaciones Hidrográficas españolas para medir la calidad o el grado de contaminación de las aguas. Es éste el índice que se emplea en la presente investigación con objeto de evaluar la calidad de las aguas superficiales circulantes por la cuenca hidrográfica del Júcar. Para su aplicación, se han considerado los datos observados sobre el conjunto de parámetros físico-químicos que definen dicho índice, en diferentes puntos de muestreo (o estaciones de control) y a lo largo de un amplio periodo de tiempo.

El análisis de la información utilizada permite evaluar la calidad de las aguas (independientemente de sus posibles utilizaciones) de la cuenca del Júcar, detectando los cambios producidos en la evolución temporal del ICG y de sus componentes. También se considera en el estudio la altitud de las estaciones de control seleccionadas, ya que dicha variable es importante a la hora de caracterizar las masas de agua superficial (tal y como se expresa en la Directiva 2000/60/CE del Parlamento Europeo y del Consejo de 23 de octubre de 2000 por la que se establece un marco comunitario de actuación en el ámbito de la política de aguas). Además, mediante la aplicación de técnicas de análisis de datos de panel, se analiza el comportamiento del ICG en función de los denominados parámetros básicos, considerando otras variables de control como el caudal circulante y variables ficticias temporales.

El trabajo se ha organizado del siguiente modo. A continuación, en la sección 2, se explica la metodología utilizada en el presente estudio. En la sección 3 se realizan algunos comentarios sobre la obtención de los datos utilizados en el trabajo. La sección 4 aborda el análisis de los resultados obtenidos. Por último, la sección 5 presenta las principales conclusiones de la investigación. 


\section{METODOLOGÍA}

\subsection{MEDICIÓN DE LA CALIDAD GENERAL DEL AGUA: EL ICG}

En este trabajo se utiliza el ICG para medir la calidad de las aguas de la cuenca del Júcar. Dicho índice, definido para evaluar la calidad del agua con independencia de su uso posterior, se puede expresar así:

$$
\mathrm{ICG}=\sum_{i=1}^{n} q_{i} p_{i}
$$

donde $n$ indica el número de parámetros (o características físico-químicas) que intervienen en el cálculo del índice; $q_{i}$ representa una función que transforma el valor observado en la muestra del parámetro i en un nivel de calidad que varía entre 0 y 100 (siendo el valor 0 el nivel pésimo y 100 el óptimo); y $p_{i}$ es la ponderación correspondiente al parámetro i-ésimo, de forma que la suma de todas las ponderaciones sea la unidad con la finalidad de que el índice de calidad oscile entre 0 y 100.

En el cálculo del ICG intervienen dos tipos de parámetros: básicos (que siempre se consideran en el cálculo del índice); y complementarios (que sólo intervienen en su cálculo cuando el valor de $q_{i}$ que le corresponda sea inferior a 60).

Para cada muestra de agua analizada se procede a calcular los correspondientes valores de las funciones $q_{i}$ y del ICG. La escala de medición establece que la calidad del agua es: excelente cuando el valor del ICG está comprendido entre 100 y 90 ; buena, si el valor oscila entre 90 y 80 ; intermedia, en el caso que el índice tome un valor entre 80 y 70 ; admisible, si el valor está entre 70 y $60 ;$ y, finalmente, inadmisible, cuando el índice toma valores entre 60 y 0 .

\subsection{ANÁLISIS DEL COMPORTAMIENTO DEL ICG EN FUNCIÓN DE SUUS PARAMETROS BASICOS: MODELO CON DATOS DE PANEL}

Una vez calculados, para cada muestra de agua, los valores de las funciones $q_{i}$ y del ICG, se obtienen sus correspondientes valores medios anuales en cada uno los puntos de muestreo seleccionados. A partir de esos valores medios anuales se estima un modelo de datos de panel con objeto de estudiar el comportamiento del ICG en función de sus parámetros básicos.

De forma general, un modelo de datos de panel puede especificarse de la siguiente forma: 


$$
y_{i t}=x_{i t}^{\prime} \beta+\alpha_{i}+u_{i t} \quad i=1, \ldots, N, \quad t=1, \ldots, \mathrm{T}
$$

donde $y_{i t}$ es la variable dependiente, que será el ICG, en la que los subíndices $i$ y $t$ hacen referencia a la estación de control y al periodo de tiempo correspondiente; $x_{i t}$ es un vector que contiene las variables explicativas del modelo, que en este caso serán los parámetros básicos que integran el índice; $\beta$ es el vector de coeficientes que se pretende estimar; $a_{i}$ recoge el efecto individual, específico para cada estación de control y que no cambia a lo largo del tiempo; $u_{i t}$ es un término de perturbación aleatoria. En $x_{i t}$ también se va a incluir el caudal de agua circulante (como variable de control) y los efectos temporales (representados por las correspondientes variables ficticias de tiempo).

La estimación del vector de coeficientes $B$ se puede efectuar mediante el enfoque de efectos fijos o mediante el de efectos aleatorios. La elección entre un modelo de efectos fijos y un modelo de efectos aleatorios suele depender de la existencia o no de correlación entre los efectos individuales y las variables explicativas. La estrategia habitual de especificación en el caso de un modelo como el representado por la ecuación anterior es la contrastación, mediante un test diseñado por Hausman, de la hipótesis nula de incorrelación entre $a_{i}$ y las variables explicativas. Si se rechaza la hipótesis nula, la aplicación de la transformación intragrupos permite obtener estimadores consistentes de los parámetros. Por el contrario, si se acepta dicha hipótesis puede obtenerse un estimador más eficiente que el intragrupos a través del método de los Mínimos Cuadrados Generalizados. En este trabajo se sigue un enfoque de efectos fijos, ya que la aplicación del test de Hausman confirma la existencia de correlación entre los efectos individuales y las variables explicativas.

\section{DATOS: ESTACIONES DE CONTROL Y PARÁMETROS}

En el ámbito de la cuenca del Júcar se ha seleccionado una muestra de 60 estaciones de control y los datos obtenidos sobre ellas cubren el periodo comprendido entre los años 2000 y 2009 (ambos inclusive). Para la realización de algunos análisis, el mencionado periodo temporal se divide en dos partes: en la primera se consideran los datos observados desde el año 2000 hasta el año 2004, y en la segunda se incluyen los datos observados entre los años 2005 y 2009.

Las estaciones de control forman parte de la Red Oficial de Control de Calidad de la Confederación Hidrográfica del Júcar, y están repartidas a lo largo de todo el territorio administrado por dicha confederación: 6 en la provincia de Teruel; 7 en la provincia de Cuenca; 5 en la provincia de 
Castellón; 24 en la provincia de Valencia; 6 en la provincia de Albacete y 12 en la provincia de Alicante.

Además, la distribución de las estaciones de control según su altitud se traduce en la existencia de 20 estaciones en cada una de las tres categorías especificadas en la Directiva Marco del Agua o Directiva 2000/60/CE (curso alto, cuando la altitud es superior a 800 metros; curso medio cuando la altitud se sitúa entre 200 y 800 metros y curso bajo, si la altitud es inferior a 200 metros).

En el Cuadro 1 se explicitan los 23 parámetros (o características físico-químicas) que componen el ICG y que han sido observados en las estaciones de control seleccionadas.

\begin{tabular}{|l|l|l|l|}
\hline \multicolumn{1}{|c|}{ Parámetro } & Denominación & Unidad de medida & \multicolumn{1}{c|}{ Tipo } \\
\hline Cadmio & cadmio & $\mathrm{mg} / \mathrm{L} \mathrm{Cd}$ & Complementario \\
\hline Calcio & calcio & $\mathrm{mg} / \mathrm{L} \mathrm{Ca}$ & Complementario \\
\hline Cianuros & cianur & $\mathrm{mg} / \mathrm{L} \mathrm{CN}$ & Complementario \\
\hline Cloruros & clorur & $\mathrm{mg} / \mathrm{L} \mathrm{Cl}$ & Complementario \\
\hline Cobre & cobre & $\mathrm{mg} / \mathrm{L} \mathrm{Cu}$ & Complementario \\
\hline Coliformes totales a $37^{\circ} \mathrm{C}$ & coltot & $\mathrm{Col} / 100 \mathrm{~mL}$ & Básico \\
\hline Conductividad & conduc & $\mathrm{mS} / \mathrm{cm} \mathrm{a} 20^{\circ} \mathrm{C}$ & Básico \\
\hline Cromo hexavalente & crhexa & $\mathrm{mg} / \mathrm{L} \mathrm{Cr}(\mathrm{VI})$ & Complementario \\
\hline Demanda bioquímica de oxígeno & dbo & $\mathrm{mg} / \mathrm{L} \mathrm{O} 2$ & Básico \\
\hline Demanda química de oxígeno & dqo & $\mathrm{mg} / \mathrm{L} \mathrm{O} 2$ & Básico \\
\hline Detergentes & deterg & $\mathrm{mg} / \mathrm{L} \mathrm{LAS}$ & Complementario \\
\hline Fenoles & fnols & $\mathrm{mg} / \mathrm{L} \mathrm{C6H5OH}$ & Complementario \\
\hline Fosfatos totales & fosfat & $\mathrm{mg} / \mathrm{L} \mathrm{PO4}$ & Básico \\
\hline Magnesio & magne & $\mathrm{mg} / \mathrm{L} \mathrm{Mg}$ & Complementario \\
\hline Materias en suspensión & solsus & $\mathrm{mg} / \mathrm{L} \mathrm{Hg}$ & Básico \\
\hline Mercurio & mercur & $\mathrm{mg} / \mathrm{L} \mathrm{Hg}$ & Complementario \\
\hline Nitratos & nitrat & $\mathrm{mg} / \mathrm{L} \mathrm{NO3}$ & Básico \\
\hline Oxígeno disuelto & oxidis & $\%$ O2 & Básico \\
\hline pH & ph & & Básico \\
\hline Plomo & plomo & $\mathrm{mg} / \mathrm{L} \mathrm{Pb}$ & Complementario \\
\hline Sodio & sodio & $\mathrm{mg} / \mathrm{L} \mathrm{Na}$ & Complementario \\
\hline Sulfatos & sulfat & $\mathrm{mg} / \mathrm{L} \mathrm{SO} 4$ & Complementario \\
\hline Zinc & zinc & Zinc & Complementario \\
\hline
\end{tabular}

Cuadro 1: Parámetros componentes del ICG

Fuente: Confederación Hidrográfica del Júcar y Ministerio de Medio Ambiente

\section{RESULTADOS}

\subsection{EVALUACIÓN DE LA CALIDAD, GENERAL DE LAS AGUAS SUUPERFICIALES EN LA CUENCA DEL JÚCAR}

Los valores del ICG obtenidos a partir de los datos permiten clasificar la calidad general de las aguas superficiales de la cuenca del Júcar. En el 
Cuadro 2 se presenta, para el conjunto de las 60 estaciones seleccionadas y para los tres grupos en los que éstas se clasifican según su altitud (baja, media y alta), el valor medio del ICG en el periodo temporal completo (2000-2009) y en cada uno de los subperiodos en que se ha dividido éste (2000-2004 y 2005-2009). En ese mismo cuadro también se muestran los niveles de significación críticos ( $p$-valores) asociados al test $t$ utilizado para comparar los valores medios del índice entre los dos subperiodos temporales mencionados.

En el Cuadro 3 se recogen los resultados correspondientes a la comparación de medias (entre los dos subperiodos temporales) para los valores de las funciones $q_{i}$ asociadas a cada uno de los parámetros que intervienen en la definición del ICG.

Considerando el periodo temporal completo, el valor promedio del ICG obtenido para el conjunto formado por todas las estaciones seleccionadas indica que la calidad general del agua, en la cuenca del Júcar, puede calificarse como intermedia (el ICG promedio es 75.77).

No obstante, el resultado anterior debe matizarse, si se considera la categorización de las estaciones según su altitud. Así, se puede observar cómo empeora la calidad del agua a medida que las estaciones de control se sitúan en altitudes más bajas. El valor medio del índice, para el periodo temporal completo, es igual a 82.01 en el conjunto de estaciones de mayor altitud (lo que representa una calidad buena) y cae hasta el valor 68.62 cuando se trata del grupo de estaciones de control situadas en altitudes bajas (lo que permite calificar la calidad de sus aguas simplemente como admisible). Realizando un análisis de la varianza, se detecta que existen diferencias estadísticamente significativas entre los niveles medios de calidad de las estaciones situadas en los tres grupos que se establecen atendiendo al criterio de la altitud (para el periodo temporal completo, el valor del estadístico $\mathrm{F}$ asociado a dicho análisis es igual a 59.46, con un nivel de significación crítico inferior a 0.001).

Observando los resultados correspondientes a la comparación de los valores medios del ICG en los dos subperiodos de tiempo en los que se descompone el periodo muestral completo, no se detecta la existencia de cambios temporales estadísticamente significativos en el comportamiento del índice para el conjunto de las estaciones de control. Sólo se observa que existen diferencias significativas en el grupo de estaciones de mayor altitud, que experimentan un empeoramiento en la calidad de sus aguas (el ICG promedio cae desde el valor 84.59 hasta el valor 79.38). En el caso de las funciones asociadas a los 23 parámetros componentes del ICG, los valores medios asociados a cianuros, demanda química de oxígeno, mercurio, plomo, sulfatos y zinc experimentan cambios temporales significativos (sus niveles de significación críticos no superan el valor 0.05), mejorando la calidad de todos ellos, excepto en el caso del zinc. De 
todos estos parámetros, únicamente la demanda química de oxígeno es considerado como un parámetro básico.

\begin{tabular}{|l|c|c|c|c|c|}
\hline & \multicolumn{3}{|c|}{ Media ICG } & \multicolumn{2}{c|}{$\begin{array}{c}\text { Comparación entre periodos } \\
2000-2004 \\
\text { y 2005-2009 }\end{array}$} \\
\hline \multicolumn{1}{|c|}{ Estaciones } & $\begin{array}{c}\text { Periodo } \\
2000-2009\end{array}$ & $\begin{array}{c}\text { Periodo } \\
2000-2004\end{array}$ & $\begin{array}{c}\text { Periodo } \\
2005-2009\end{array}$ & Diferencia & p-valor \\
\hline Todas & 75.77 & 76.42 & 75.11 & 1.31 & 0.173 \\
\hline Altitud baja & 68.62 & 67.82 & 69.42 & -1.60 & 0.426 \\
\hline Altitud media & 76.86 & 77.46 & 76.27 & 1.19 & 0.337 \\
\hline Altitud alta & 82.01 & 84.59 & 79.38 & 5.21 & 0.000 \\
\hline
\end{tabular}

Cuadro 2: Valores medios del ICG, para todas las estaciones y según su altitud, en los distintos periodos de tiempo

\begin{tabular}{|l|c|c|c|c|c|}
\hline \multirow{2}{*}{ Parámetro } & \multicolumn{3}{|c|}{ Media de las funciones } & $q_{i}$ & \multicolumn{2}{c|}{$\begin{array}{c}\text { Comparación entre periodos } \\
\mathbf{2 0 0 0 - 2 0 0 4} \text { y 2005-2009 }\end{array}$} \\
\hline & $\begin{array}{c}\text { Periodo } \\
\mathbf{2 0 0 0 - 2 0 0 9}\end{array}$ & $\begin{array}{c}\text { Periodo } \\
\mathbf{2 0 0 0 - 2 0 0 4}\end{array}$ & $\begin{array}{c}\text { Periodo } \\
\mathbf{2 0 0 5 - 2 0 0 9}\end{array}$ & Diferencia & p-valor \\
\hline cadmio & 99.92 & 99.95 & 99.88 & 0.07 & 0.413 \\
\hline calcio & 94.56 & 93.71 & 95.27 & -1.56 & 0.217 \\
\hline cianur & 99.46 & 98.96 & 100 & -1.04 & 0.009 \\
\hline clorur & 83.41 & 82.24 & 84.38 & -2.14 & 0.345 \\
\hline coltot & 32.92 & 33.04 & 32.84 & 0.20 & 0.943 \\
\hline conduc & 45.64 & 45.78 & 45.50 & 0.28 & 0.894 \\
\hline crhexa & 99.94 & 99.95 & 99.94 & 0.01 & 0.837 \\
\hline dbo & 89.67 & 89.00 & 90.35 & -1.35 & 0.348 \\
\hline dqo & 70.98 & 62.09 & 77.39 & -15.30 & 0.000 \\
\hline deterg & 97.37 & 96.69 & 98.06 & -1.37 & 0.145 \\
\hline fnols & 99.36 & 99.43 & 99.28 & 0.15 & 0.726 \\
\hline fosfat & 92.44 & 92.05 & 92.74 & -0.69 & 0.647 \\
\hline magne & 85.53 & 84.77 & 86.16 & -1.39 & 0.454 \\
\hline solsus & 97.20 & 96.89 & 97.52 & -0.63 & 0.295 \\
\hline mercur & 97.99 & 96.12 & 99.97 & -3.85 & 0.000 \\
\hline nitrat & 95.59 & 95.58 & 95.59 & -0.01 & 0.979 \\
\hline oxidis & 93.84 & 92.96 & 94.72 & -1.76 & 0.121 \\
\hline ph & 77.53 & 77.55 & 77.52 & 0.03 & 0.963 \\
\hline plomo & 99.76 & 99.68 & 99.86 & -0.18 & 0.026 \\
\hline sodio & 78.54 & 77.38 & 79.50 & -2.12 & 0.387 \\
\hline sulfat & 76.46 & 74.57 & 78.05 & -3.48 & 0.050 \\
\hline zinc & 99.59 & 99.87 & 99.32 & 0.55 & 0.000 \\
\hline Cuadr & & & & & \\
\hline
\end{tabular}

Cuadro 3: Valores medios de las funciones de los parámetros del ICG, para todas las estaciones, en los distintos periodos de tiempo

Además. tras realizar los contrastes necesarios para comparar los valores medios del ICG en los dos subperiodos temporales para cada una de las 60 estaciones de control seleccionadas, resulta que en 23 estaciones se detectan cambios temporales significativos en el comportamiento 
del ICG (con niveles críticos de significación inferiores al 5\%), de forma que en 15 de esas estaciones la calidad del agua empeora y en los otros 8 casos la calidad experimenta una mejoría.

\subsection{COMPORTAMIENTO DEL ICG EN FUNCIÓN DE SUS PARÁME- TROS BASICOS}

Otro aspecto abordado en este trabajo es el estudio de la influencia que sobre el ICG tienen los parámetros básicos que lo integran (coliformes totales, conductividad, demanda bioquímica de oxígeno, demanda química de oxígeno, fosfatos totales, materias en suspensión, nitratos, oxígeno disuelto y ph), aplicando técnicas de análisis de datos de panel. Esta metodología permite controlar el efecto individual específico de cada uno de los puntos de muestreo considerados en el estudio.

Con los valores medios anuales del ICG y de las funciones de los parámetros básicos, en las 60 estaciones de control seleccionadas, para los 10 años comprendidos en el periodo 2000-2009, se ha obtenido el panel de datos necesarios para estimar el modelo lineal que explica el comportamiento del índice en función de los mencionados parámetros (un análisis previo de correlaciones bivariantes, revela existencia de relaciones lineales significativas entre el ICG y cada uno de esos parámetros).

Sustituyendo en la ecuación [1] la variable dependiente por el ICG, el vector de variables explicativas por los parámetros básicos e incluyendo el caudal circulante por las estaciones como variable de control, el modelo a estimar se puede especificar de la siguiente forma:

$$
\begin{aligned}
I C G_{i t}= & \beta_{0}+\beta_{1} \text { caudal }_{i t}+\beta_{2} \text { coltot }_{i t}+\beta_{3} \text { conduc }_{i t}+\beta_{4} \text { dbo }_{i t}+\beta_{5} \text { dqo }_{i t}+ \\
& +\beta_{6} \text { fosfat }_{i t}+\beta_{7} \text { solsus }_{i t}+\beta_{8} \text { nitrat }_{i t}+\beta_{9} \text { oxidis }_{i t}+\beta_{10} \text { ph }_{i t}+\alpha_{i}+u_{i t}
\end{aligned}
$$

para

$$
i=1, \ldots, 60, \quad t=1, \ldots, 10
$$

En el Cuadro 4 se presentan algunos de los resultados obtenidos en la estimación del modelo, con todos los datos del panel (periodo 2000-2009) y con cada uno de los dos conjuntos que resultan al considerar los dos subperiodos temporales (2000-2004 y 2005-2009).

Las estimaciones de los coeficientes asociados a las variables explicativas del modelo se han obtenido mediante la aplicación de la transformación intragrupos. Se ha seguido este enfoque de efectos fijos ya que el resultado del test de Hausman conduce al rechazo de la hipótesis de que no existe correlación entre los efectos individuales y las variables explicativas. Además, en el modelo se incluyen variables ficticias temporales. 
Los resultados referentes a capacidad explicativa y significatividad global del modelo ( $R^{2}$ y test $F$ ) evidencian, como cabía esperar, el alto grado de representatividad del mismo. En cuanto al análisis del efecto que tienen sobre el ICG sus parámetros físico-químicos básicos individualmente considerados, las estimaciones y los p-valores de los coeficientes asociados a dichos parámetros permiten afirmar que todos $\mathrm{y}$ cada uno de ellos tienen una influencia positiva y estadísticamente significativa sobre el ICG. Sólo los nitratos, cuando se estima el modelo con los datos del periodo 2000-2004, y los fosfatos totales, en el modelo estimado con los datos del periodo 2005-2009, parecen no tener una influencia significativa sobre el ICG.

\begin{tabular}{|c|c|c|c|}
\hline \multicolumn{4}{|c|}{ Variable dependiente: ICG } \\
\hline \multirow[b]{2}{*}{ Variable explicativa } & \multicolumn{3}{|c|}{ Coeficiente (p-valor) } \\
\hline & $\begin{array}{c}\text { Periodo } \\
\text { 2000-2009 }\end{array}$ & $\begin{array}{c}\text { Periodo } \\
\text { 2000-2004 }\end{array}$ & $\begin{array}{c}\text { Periodo } \\
\text { 2005-2009 }\end{array}$ \\
\hline Caudal & $0.0356(0.457)$ & $0.0386(0.566)$ & $0.0330(0.627)$ \\
\hline Coliformes totales a $37^{\circ} \mathrm{C}$ & $0.1130(0.000)$ & $0.1112(0.000)$ & $0.1282(0.000)$ \\
\hline Conductividad & $0.1936(0.000)$ & $0.1335(0.001)$ & $0.1765(0.000)$ \\
\hline Demanda bioquímica de oxígeno & $0.1192(0.000)$ & $0.1091(0.000)$ & $0.1155(0.000)$ \\
\hline Demanda química de oxígeno & $0.0334(0.001)$ & $0.0203(0.000)$ & $0.0378(0.013)$ \\
\hline Fosfatos totales & $0.0434(0.002)$ & $0.0615(0.000)$ & $0.0609(0.254)$ \\
\hline Materias en suspensión & $0.1827(0.000)$ & $0.1663(0.000)$ & $0.1803(0.000)$ \\
\hline Nitratos & $0.1477(0.000)$ & $-0.0076(0.903)$ & $0.1909(0.017)$ \\
\hline Oxígeno disuelto & $0.1612(0.000)$ & $0.1402(0.000)$ & $0.1402(0.001)$ \\
\hline $\mathrm{pH}$ & $0.1667(0.000)$ & $0.1568(0.001)$ & $0.1103(0.016)$ \\
\hline $\mathrm{R}^{2}$ within & 0.7972 & 0.8651 & 0.8158 \\
\hline Test $\mathrm{F}$ significatividad global & $90.71(0.000)$ & $112.57(0.000)$ & $93.81(0.000)$ \\
\hline Test $\mathrm{F}$ parámetros básicos & $145.25(0.000)$ & $109.29(0.000)$ & $99.03(0.000)$ \\
\hline Test $\mathrm{F}$ variables temporales & $4.40(0.000)$ & $3.47(0.010)$ & $3.46(0.010)$ \\
\hline
\end{tabular}

Cuadro 4: Comportamiento del ICG en función de sus parámetros básicos, en el periodo global (2000-2009) y en los subperiodos (2000-2004 y 2005-2009)

Nota: Los resultados de estimación del modelo se han obtenido siguiendo un enfoque de efectos fijos, mediante la aplicación de la transformación intragrupos, de forma robusta e incluyendo variables ficticias temporales

Por otra parte, en el Cuadro 5 se recogen los resultados de estimación del modelo si se consideran, por separado, los tres conjuntos de estaciones resultantes cuando se clasifican según su altitud (baja, media y alta). Se observa que, en el grupo de estaciones de altitud media (entre 200 y 800 metros), todos los parámetros básicos presen- 
tan una influencia positiva y significativa sobre el ICG. Sin embargo, en el conjunto de estaciones de menor altitud, los fosfatos totales, los nitratos y el $p h$ no manifiestan un efecto significativo sobre el índice general, mientras que para el grupo de estaciones de mayor altitud los parámetros que no muestran una influencia estadísticamente significativa sobre el ICG son la demanda química de oxígeno, los fosfatos totales, los nitratos y el oxígeno disuelto.

Por lo que se refiere a la variable caudal, en ninguno de los escenarios contemplados, parece tener un impacto determinante sobre el ICG (los p-valores o niveles críticos de significación son elevados en todos los casos).

\begin{tabular}{|l|c|c|c|}
\hline \multicolumn{4}{|c|}{ Variable dependiente: ICG } \\
\hline \multirow{2}{*}{ Variable explicativa } & Altitud baja & Altitud media & Altitud alta \\
\cline { 2 - 4 } & $0.0918(0.466)$ & $-0.0392(0.642)$ & $-0.0549(0.375)$ \\
\hline Caudal & $0.1059(0.008)$ & $0.1196(0.000)$ & $0.1046(0.000)$ \\
\hline Coliformes totales a $37^{\circ} \mathrm{C}$ & $0.2758(0.009)$ & $0.1578(0.000)$ & $0.1681(0.000)$ \\
\hline Demductividad & $0.0948(0.014)$ & $0.1254(0.000)$ & $0.1533(0.000)$ \\
\hline Demanda bioquímica de oxígeno & $0.0491(0.078)$ & $0.0299(0.043)$ & $0.0169(0.441)$ \\
\hline Fosfatos totales & $0.0066(0.810)$ & $0.0900(0.000)$ & $-0.3704(0.437)$ \\
\hline Materias en suspensión & $0.2052(0.000)$ & $0.1759(0.000)$ & $0.1816(0.000)$ \\
\hline Nitratos & $0.1351(0.241)$ & $0.2512(0.000)$ & $0.0734(0.831)$ \\
\hline Oxígeno disuelto & $0.1831(0.000)$ & $0.1231(0.000)$ & $0.1868(0.338)$ \\
\hline pH & $0.1908(0.203)$ & $0.1206(0.010)$ & $0.2128(0.000)$ \\
\hline $\mathrm{R}^{2}$ within & 0.8815 & 0.7510 & 0.8722 \\
\hline Test F significatividad global & $31.93(0.000)$ & $48.37(0.000)$ & $77.39(0.000)$ \\
\hline Test F parámetros básicos & $47.24(0.000)$ & $74.63(0.000)$ & $80.51(0.000)$ \\
\hline Test F variables temporales & $1.17(0.335)$ & $1.74(0.083)$ & $4.56(0.001)$ \\
\hline
\end{tabular}

Cuadro 5: Comportamiento del ICG en función de sus parámetros básicos, en el periodo global, para las estaciones clasificadas según su altitud

Nota: Los resultados de estimación del modelo se han obtenido siguiendo un enfoque de efectos fijos, mediante la aplicación de la transformación intragrupos, de forma robusta e incluyendo variables ficticias temporales

\section{CONCLUSIONES}

En este trabajo se analiza la calidad general del agua superficial en el ámbito de la cuenca del Júcar, a lo largo del periodo 2000-2009. Se determinan los valores del denominado Índice de Calidad General (ICG), en un conjunto de 60 de puntos de muestreo y en distintos momentos del periodo temporal indicado, a partir de los datos observados sobre los 23 parámetros físico-químicos que definen el citado índice.

Considerando el periodo temporal completo (2000-2009), el valor promedio del ICG obtenido para el conjunto formado por todas las estacio- 
nes seleccionadas indica que la calidad general del agua, en la cuenca del Júcar, puede calificarse como intermedia.

En el estudio también se ha tenido en cuenta la altitud correspondiente a las estaciones de control seleccionadas. Al clasificar las estaciones en tres categorías según su altitud, se observa que la calidad del agua no es la misma en los tres grupos, de forma que empeora a medida que las estaciones de control se sitúan en altitudes más bajas.

El análisis comparativo de los valores medios del ICG en los dos subperiodos de tiempo en los que se ha dividido el periodo muestral completo, permite concluir que no se producen cambios temporales estadísticamente significativos en el comportamiento del índice para el conjunto de las estaciones de control. Sólo se observa que existen diferencias significativas en el grupo de estaciones de mayor altitud, que experimentan un empeoramiento en la calidad de sus aguas. De todos los parámetros que definen el ICG, los valores medios asociados a cianuros, demanda química de oxígeno, mercurio, plomo, sulfatos y zinc son los que evidencian cambios temporales significativos, mejorando la calidad de todos ellos, excepto en el caso del zinc.

En el trabajo también se estudia, mediante la estimación de un modelo de datos de panel, la influencia que tienen sobre el ICG los parámetros básicos que lo integran (coliformes totales, conductividad, demanda bioquímica de oxígeno, demanda química de oxígeno, fosfatos totales, materias en suspensión, nitratos, oxígeno disuelto y ph). El modelo especificado permite controlar el efecto individual específico de cada una de las estaciones de medición seleccionadas en la investigación y también considera la influencia del caudal de agua circulante por las mismas.

Los resultados de estimación del modelo revelan su alto grado de capacidad explicativa. En cuanto al análisis del efecto que tienen sobre el ICG sus parámetros físico-químicos básicos individualmente considerados, los resultados permiten afirmar que todos y cada uno de ellos tienen una influencia positiva y estadísticamente significativa sobre el ICG en el periodo temporal completo (2000-2009). Cuando se estima el modelo, por separado, con los datos de cada uno de los dos subperiodos temporales (2000-2004 y 2005-2009), se evidencian pocas excepciones respecto a este comportamiento.

Por otra parte, al clasificar las estaciones según su altitud, los resultados de estimación indican que todos los parámetros básicos presentan una influencia positiva y significativa sobre el ICG en el caso de considerar las estaciones de altitud media. Sin embargo, en el conjunto de estaciones de menor altitud, los fosfatos totales, los nitratos y el ph no manifiestan un efecto significativo sobre el índice general, mientras que para el grupo de estaciones de mayor altitud los parámetros que no muestran una influencia estadísticamente significativa sobre el ICG son la demanda química de oxígeno, los fosfatos totales, los nitratos y el oxígeno disuelto. 
Por último, en ninguno de los casos analizados, el caudal de agua circulante por las estaciones de control evidencia tener un impacto significativo sobre el ICG.

\section{BIBLIOGRAFÍA}

Barberán, R.; Costa, A. y Alegre, A. (2008): "Los costes de los servicios urbanos del agua. Un análisis necesario para el establecimiento y control de tarifas". Hacienda Pública Española / Revista de Economía Pública, 186: pp. 123-155.

Barreiro, J. y Pérez, L. (2006): "Beneficios sociales en la mejora de la calidad del agua: una aproximación a partir de los costes defensivos de los hogares". Estudios de Economía Aplicada, 24-1: pp. 453-476.

Beamonte, E.; Bermúdez, J.; Casino, A. y Veres, E. (2004): "La calidad del agua en ciertas estaciones de control del canal Júcar-Turia (periodo 1994-2001)". Revista Española de Estudios Agrosociales y Pesqueros, 201: pp. 105-126.

Beamonte, E.; Bermúdez, J.; Casino, A. y Veres, E. (2005): "A global stochastic index for water quality: the case of the river Turia (Spain)". Journal of Agricultural, Biological and Environmental Statistics, 10: pp. 424-439. Beamonte, E.; Bermúdez, J.; Casino, A. y Veres, E. (2007): "A statistical study of the quality of surface water intended for human consumption near Valencia (Spain)". Journal of Environmental Management, 83: pp. 307-314. Beamonte, E.; Casino, A. y Veres, E. (2010): "Water quality indicators: Comparison of a probabilistic index and a general quality index. The case of the Confederación Hidrográfica del Júcar (Spain)". Ecological Indicators, 10: pp. 1049-1054.

Bergstrom, J. C.; Boyle, K. J. y Poe, G. L. (2001): The Economic Value of Water Quality. Edward Elgar Publishers, Northampton.

González, F. (2005): "El precio del agua en las ciudades. Reflexiones y recomendaciones a partir de la Directiva 2000/60/CE". Ciudad y Territorio. Estudios territoriales, XXXVII-144: pp. 305-320.

Júdez, L.; Ibáñez, M.; Pérez Ugalde, C.; De Andrés, R.; Urzainqui, E. y Fuentes-pila, J. (2001): "Valoración del uso recreativo de un humedal español. Tests y comparación de diferentes métodos de valoración". Revista Española de Estudios Agrosociales y Pesqueros, 201: pp. 83-104. Otto, D. y Holtkamp, J. (1999): "Valuation of Water Quality in Livestock Regions: An Application to Rural Watersheds in Iowa". Journal of Agricultural \& Applied economics, 31 (1): pp. 177-184.

Pérez, C.D.; Gómez, C.M. y Garrido, R. (2010): "Cambio estructural regional y agua: escasez, dependencia e impactos sobre el tejido económico". Estudios de Economía Aplicada, 28-2: pp. 423-446. 
Prat, N. y Munné, A. (2000): "Water use and quality and stream flow in a Mediterranean stream". Water Research, 34: pp. 3876-3881.

Ribaudo, M. O. (2003): "The Economic Value of water Quality". Agricultural Economics, 29 (2): pp. 235-236.

Sánchez, E.; Colmenarejo, M.F.; Vicente, J.; Rubio, A.; García, M.G.; Travieso, L. y Borja, R. (2007): "Use of the water quality index and dissolved oxygen deficit as simple indicators of watersheds pollution". Ecological Indicators, 7: pp. 315-328.

Sevilla, M.; Torregrosa, T. y Moreno, L. (2010): "Un panorama sobre la economía del agua". Estudios de Economía Aplicada, 28-2: pp. 265-304. Steinnes, D. N. (1992): "Measuring the Economic Value of Water Quality: The Case of Lakeshore Land". The Annals of Regional Science, 26 (2): pp. 171-176. Villalba, M.; Barragán, F. J., Ternero, M. y Jiménez, J. C. (1995): "Hydrochemical study of an aquifer system in an agricultural area in South West Spain". Water Research, 29 (5): pp. 1361-1372.

\section{DATOS ACADÉMICOS}

\section{Eduardo Beamonte Córdoba}

Fecha y lugar de nacimiento: 07-07-1965 (Zaragoza)

Formación Académica: Doctor en Matemáticas (Departamento de Estadística e Investigación Operativa Aplicada), Universidad de Valencia. Experiencia Docente: Profesor de la Universidad de Valencia (Departamento de Economía Aplicada) desde 1990.

Situación profesional actual: Profesor Titular de Universidad en el Departamento de Economía Aplicada de la Universidad de Valencia

\section{Alejandro Casino Martínez}

Fecha y lugar de nacimiento: 30-03-1965 (Valencia)

Formación Académica: Doctor en Ciencias Económicas y Empresariales (Departamento de Economía Aplicada), Facultad de Economía (Universidad de Valencia).

Experiencia Docente: Profesor de la Universidad de Valencia (Departamento de Economía Aplicada) desde 1988.

Situación profesional actual: Profesor Catedrático de Escuela Universitaria en el Departamento de Economía Aplicada de la Universidad de Valencia

\section{Ernesto Jesús Veres Ferrer}

Fecha y lugar de nacimiento: 30-12-1952 (Valencia)

Formación Académica: Doctor en Ciencias Exactas, Universidad de Valencia. 
Experiencia Profesional: Estadístico Facultativo del Instituto Nacional de Estadística, Delegado de Estadística en Teruel, Alicante y Castellón, Director Adjunto del Instituto Valenciano de Estadística y Jefe del Servicio de Coordinación del Ministerio de Fomento.

Experiencia Docente: Profesor de las Universidades Autónoma de Madrid, Zaragoza y UNED. Profesor de la Universidad de Valencia (Departamento de Economía Aplicada) desde 1996.

Situación profesional actual: Profesor Titular de Universidad en el Departamento de Economía Aplicada de la Universidad de Valencia

\section{ARTÍCULOS DE INVESTIGACIÓN MÁS RELEVANTES EN RELA- CIÓN CON EL MEDIOAMBIENTE (BEAMONTE, CASINO Y VERES):}

(2010): Water quality indicators: comparison of a probabilistic index and a general quality index. The case of the Confederación Hidrográfica del Júcar (Spain), Ecological Indicators, 10, pp. 1049-1054.

(2007): A statistical study of the quality of surface water intended for human consumption near Valencia, Journal of Environmental Management, Elseiver, 83, pp.307-314.

(2006): Diseño de un índice para la medición de la calidad fisicoquímica del agua piscícola. Aplicación a la cuenca hidrográfica del Jucar. Revista Española de Estudios Agrosociales y Pesqueros, 209, pp. 215-232.

(2005): A Global Stochastic Index for Water Quality: The Case of The River Turia in Spain, Journal of Agricultural, Biological and Environmental Statistics, 10 (4), pp. 424-439.

(2004): La calidad del agua en ciertas estaciones de control del canal Júcar-Turia (período 1994-2001), Revista Española de Estudios Agrosociales y Pesqueros, 201, pp. 105-126.

(2004): Un indicador global para la calidad del agua. Aplicación a las aguas superficiales de la Comunidad Valenciana, Revista Estadística Española, 46, número 156, pp. 357-384. 